\title{
The Value of Routine Radiology in Young Psychiatric Patients
}

\author{
Major N Raby \\ MRCP, FRCR, RAMC, Senior Specialist \\ Dept of Radiology, Queen Elizabeth Military Hospital, Woolwich, London SE184QH
} SUMMARY: The current policy of obtaining routine skull and chest X-rays on all psychiatric admissions to military
hospitals has been examined by scrutiny of the radiology reports on all such admissions during one calendar year. No
clinically unexpected finding was demonstrated in any of the 352 patients so examined. Routine radiological investigation
of these patients may safely be abandoned.

\section{Introduction}

The Queen Elizabeth Military Hospital is the main psychiatric referral centre for the Army in the UK and it also provides a service to the local civilian community. It is the policy of the psychiatric division routinely to request chest and lateral skull $\mathrm{X}$-rays on all inpatients. Although previous studies have indicated that such a policy is not necessary it has been suggested that selected groups such as immigrants and alcohol abusers may merit a chest film ${ }^{1}$. As a large proportion of psychiatric admissions to this hospital are alcohol related, the adopted policy may be justified. This study seeks to determine whether this is the case or not.

\section{Patients, Methods and Results}

From the computerised X-ray report file, the reports of all psychiatric inpatients for the period 1 October 1985 to 30 September 1986 were obtained. Three hundred and fifty-two patients ( 35 female, 317 male) had both chest and lateral skull X-rays performed, although the age range was $20-84$ years. Ninety percent were aged $20-45$ years, in keeping with the expected age profile of the Services. Two hundred and forty-nine were admitted to the alcohol treatment unit.

Five $(1.3 \%)$ lateral skull films were reported as showing minor calcification in the region of the pituitary fossa; coned views were recommended but no further films were requested by the referring clinician.

Six $(1.4 \%)$ chest $X$-rays were reported as showing an abnormality. In 5 of these the abnormality was already known to the referring clinician either from previous $\mathrm{X}$ rays or from the clinical history; these included 1 fractured clavicle, 1 fractured vertebral body, 1 case of cardiac failure, and 2 cases of emphysema.

One patient had a mass on the chest film which subsequently proved to be a bronchogenic carcinoma.

\section{Discussion}

No significant abnormality was detected on any lateral skull film. This accords with previously published series $^{2,3}$, and this supports the view that skull $\mathrm{X}$-rays can safely be discontinued as a routine screening investigation in service patients.

Only 1 chest X-ray disclosed a previously unknown abnormality but this patient had a 20 year history of alcohol abuse, and examination of the case notes revealed that at the time of admission there were abnormal clinical signs and a history of physical illness. The chest $X$-ray could not therefore be regarded as routine.

The results of this series accord with the conclusions of previous authors that routine chest radiology in young psychiatric patients is unnecessary ${ }^{2}$. It has beenco suggested by Hughes and Barraclough ${ }^{1}$ that patients $\mathbb{\mathscr { C }}$ with a history of alcohol abuse may represent a group $\stackrel{+}{\rightarrow}$ who should have a chest film because of the high incidence of associated disease - particularly tuber- $\frac{\vec{D}}{\mathbb{D}} \frac{T}{D}$ culosis. This study does not support that recommenda- $\stackrel{\bigcirc}{\square}$ tion for patients seen at the Queen Elizabeth Military@ Hospital. Approximately $70 \%$ of the patients studied were admitted to the alcohol treatment unit, and there $\varnothing$ were no more chest film abnormalities in this group thang those admitted for illness which was not alcohol related.

In conclusion, this study appears to show that for? young psychiatric patients admitted to service hospitals, there is no indication for routine skull or chest radiology even if there is a history of alcohol abuse.

\section{REFERENCES}

1. Hughes J and BARRAClOUGH B M. Value of routine chest radiology of psychiatric patients. $\mathrm{Br} \mathrm{Med} J 1980 ; 281$ : 1461-1462.

2. LARKIN E P. The X-ray department and psychiatry. $\mathrm{Br} J$ Psychiatry 1985; 146: 62-65.

3. RASTOGi S C and BARRACLOUGH B M. Skull radiology in patients with psychiatric illness. Br Med J 1983; 287: 1259. 